\title{
Crise de la poésie?
}

\section{Michel Decaudin}

$A$

vec une centaine d'années de recul, nous nous représentons assez bien la poésie de l'époque symboliste, ses tenants et ses aboutissants, ses courants, ses enjeux, ses sommets. Sans adopter les querelles et les jugements des contemporains, nous les situons dans un paysage où les arbres, loin de cacher la forêt, lui donnent ses couleurs et son relief.

Je me demande en revanche ce que le critique ou l'historien de la prochaine fin de siècle, à supposer qu'il y ait encore des critiques et des historiens aux abords du quatrième millénaire, pourra voir et dire de notre poésie. Il est vraisemblable que même l'aide, si performante soit-elle, d'une informatique omnisciente ne lui serait que de peu de secours pour mettre de l'ordre dans une production aussi abondante que dispersée.

Il commencerait sans doute par constater que notre seconde moitié de siècle n'a pas connu de vague de fond poétique analogue à ce que furent le Romantisme, le Symbolisme, et même le Parnasse, et que les grands courants qui l'ont traversée - l'Existentialisme, ou du moins ce qu'on a appelé de ce nom, le Nouveau Roman - ont été indifférents à la poésie ou, comme les différentes chapelles structuralistes, l'ont réduite à une simple fonction langagière.

Pas d'école poétique. Au lendemain de la guerre, le Lettrisme, la poésie "nationale" issue de la Résistance, la résurgence surréaliste qui se disait "révolutionnaire," le groupe de Rochefort ont fait long feu. Et ensuite, quoi? L'OU.LI.PO., même s'il a réuni surtout des poètes, n'est pas une entreprise exclusivement poétique. Des rapprochements autour d'une revue comme La Tour de feu à Jarnac, ou de manifestations comme les prix Arnaud et Voronca à Rodez, même si, à l'origine, lls étaient fondés sur un accord ou au moins une convergence esthétique, n'ont durê qu'en devenant insensiblement des réunions amicales.

Mais notre observateur de l'an 3000 ne manquerait pas aussi de constater que cette inexistence organique de mouvements poétiques ne signifie pas un effacement de la vie et de la création poétiques. Il relèverait les titres de dizaines, de centaines de revues, de milliers de recueils. Il n'aurait pas de peine à isoler quelques grandes oeuvres, comparables en nombre et en qualité à la production d'une période analogue du passé. Peut-être serait-il surpris de ce que la poésie semble irradier hors des formes poétiques et se répandre dans le théâtre et le roman en considérant par exemple l'oeuvre dramatique de Georges Schéhadé ou l'évolution de Michel Butor à partir de Mobile.

Généreuse et invertébrée, telle paraît bien la poésie contemporaine. Tente-t-on de la définir par une formule, la formule contraire s'impose aussitôt. Avec des oeuvres aussi différentes que celles de Ponge, de Michaux, de Guillevic, de Roubaud, de Gaspar, on entrevoit une ligne de force de poésie a-lyrique; mais tout aussi vraie est la permanence du lyrisme, de Pierre Emmanuel ou Jean-Claude Renard à Pierre Oster ou Luis Guillaume jusqu'à Philippe Delaveau. La concision de Char et de ses imitateurs incite-t-elle à penser à une poésie de l'aphorisme que viennent à l'esprit des exemples de sollicitation rhétorique chez Jean Ristat et 
d'autres. Le prolifération du vers libre et de la prose en poésie, les dispositions typographique en usage à Tel Quel évoquent un langage éclaté que contredit la prosodie d'Yves Bonnefoy. Et, à leur manière, Jean Tardieu comme Raymond Queneau illustrent cette contradiction dans des poèmes dont la structure est à la fois respect et destruction des formes traditionnelles.

Il faut ladmettre. Les efforts de classement des manuels de littérature (le chapitre "La Poésie" dans Du Surréalisme à l'empire de la critique de Germaine Brée et Edouard Morot-Sir, neuvième tome de la Littérature française de chez Arnaud, en est un bel exemple), les panoramas de la poésie donnés par Serge Brindeau ou Robert Sabatier n'aboutissent qu'à des énumérations artificiellement divisées en chapitres. La poésie des dernières décennies est le falt de créateurs solitaires, non repliês sur eux-mêmes ou enfermés dans une "tour d'tvoire," souvent au contraire très présents au monde, mais ayant entre eux peu ou pas de relations esthétiques.

Et sans disciples. Si Ponge a séduit Tel Queh, mais ne lui a inspiré que des considérations théoriques, si la concision des dernières oeuvres de Char a eu des suiveurs, souvent plus impuissants qu'exigeants, c'est en général à des modèles plus lointains que s'adressent les jeunes poètes. Alors que les jeunes symbolistes trouvaient leurs maîtres en Verlaine et Mallarmé, à peine leurs aînés de vingt ans, on se tourne vers les poèmes de Cendrars, vers les subversions de Dada, vers celles du Lettrisme ou de Cobra, vers l'OU.LI.PO qui, rappelons-le, est plus que trentenaire, les jeux de Tardieu. Phénomène général, d'ailleurs: pensons à la situation faite aujourd'hui à Henri Pichette et constatons qu'il aura fallu attendre 1994 pour l'oeuvre complète de Césaire.

Ces recours sont significatifs. Ils se réfèrent à une mise en question du contenu comme de la forme poétique et du rôle de l'auteur. Ils sont le symptôme d'un désarrol en même temps que d'une attente (que tente de combler un retour au discours lyrique).

Michel Deguy a fait une bonne analyse de cet état de crise. J'ai déjà eu l'occasion de la citer, ${ }^{1}$ mais je ne crois pas inutile de la reprendre ici:

Oui, crise profonde, bien sûr: et "théorique," et "pratique," et...*poiétique," blen sûr. Et sans précédent. La description en serait longue. Le constat de la désertion, de la "défonction" de la poésie d'un point de vue sociologique ne serait sans doute pas inutile; mais demeurerait superficiel. Ni l'optimisme à la Claude Roy ni l'indifférence méprisante à la Quignard ou à la Sollers ne permettent de prendre la mesure du phénomène - encore moins de son essence. Non seulement le sol se dérobe sous les pieds de Ladite (qu'est-ce qu' "habiter poétiquement," etc.) mais son étoffe, fouillée et dépoulllée, la laisse encore plus squelettique que nue; sans rimes ni raisons, privée de toutes les itèrations en quol elle consistait (isotopiques, rythmiques, etc.). Certes, elle n'est pas la seule à être sans umonde," mais ça n'arrange pas sa solitude, ni celle des autres. Et ce n'est pas le retour (à des régularités, prosodiques ou thématiques) quil lui donnera de retrouver ni un site, ni un "public," ni, etc. Elle manque de références comme une servante inutile. Il est vrai que le chômage est devenu une situation sociale, mais... il faut repenser tout ça (op. cit. p. 110-111). 
Un manque de références qui n'est qu'un aspect d'un phénomène plus vaste qui ronge notre société; mais cela est une autre histoire... Cette crise est-elle sans issue? N'allons pas crier précipitamment à la mort de la poésie. Parti de cette constatation qu'ailleurs il a exprimé dans la fameuse formule "On a touché au vers," Mallarmé termine "Crise de vers" par une exaltation de la poésie, et nécessité irremplaçable. N'en sera-t-il pas ainsi de la poésie, et les incertitudes, les négations ne contiennent-elles pas la promesse d'un avenir?

Il faudrait ici prendre en compte cette nouveauté du demi-siècle qu'est l'épanouissement de la francophonie et le rayonnement d'une poésie surtout africaine et canadienne, libérée des complexes hexagonaux.

Et se rappeler les derniers mots de "L'Ancien tailleur" où, en 1911, Apollinaire termine ainsi une discussion avec un interlocuteur qui ne voit rien de bon dans la poésie de son temps:

- Il se peut, dis-je à cet homme, que vous n'ayez pas entièrement tort. Mais il $\mathrm{y}$ a eu de vrais poètes après Baudelaire, et je suls certain qu'll y en a encore mais on ne les écoute guère.

- Lenas nomes?

- Je vous les diral une autre fols.

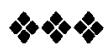

Note

${ }^{1}$ Dans mon article "Situation de la poésie française", Ibis (cahiers du séminaire "Poésie" de l'Université de Bologne), $\mathrm{n}^{\circ}$ 1, février 1994. Le texte de Michel Deguy est emprunté au livre de Bruno Grégoire, Poésies aujourd'hui (Seghers, 1990). 